\title{
Giant pop-ins and amorphization in germanium during indentation
}

\author{
David J. Oliver, ${ }^{\text {a) }}$ Jodie E. Bradby, and Jim S. Williams \\ Department of Electronic Materials Engineering, Research School of Physical Sciences and Engineering, \\ The Australian National University, Canberra, ACT 0200, Australia \\ Michael V. Swain \\ Biomaterials Science Research Unit, Faculty of Dentistry, The University of Sydney, Eveleigh, NSW 1430, \\ Australia \\ Paul Munroe \\ Electron Microscope Unit, University of New South Wales, Sydney, NSW 2052, Australia
}

(Received 19 September 2006; accepted 26 December 2006; published online 28 February 2007)

\begin{abstract}
Sudden excursions of unusually large magnitude $(>1 \mu \mathrm{m})$, "giant pop-ins," have been observed in the force-displacement curve for high load indentation of crystalline germanium $(\mathrm{Ge})$. A range of techniques including Raman microspectroscopy, focused ion-beam cross sectioning, and transmission electron microscopy, are applied to study this phenomenon. Amorphous material is observed in residual indents following the giant pop-in. The giant pop-in is shown to be a material removal event, triggered by the development of shallow lateral cracks adjacent to the indent. Enhanced depth recovery, or "elbowing," observed in the force-displacement curve following the giant pop-in is explained in terms of a compliant response of plates of material around the indent detached by lateral cracking. The possible causes of amorphization are discussed, and the implications in light of earlier indentation studies of Ge are considered. (C) 2007 American Institute of Physics. [DOI: 10.1063/1.2490563]
\end{abstract}

\section{INTRODUCTION}

From diamond-anvil cell studies it is well known that tetrahedral semiconductors undergo a structural phase transformation at elevated hydrostatic pressures. ${ }^{1}$ This results in a denser, sixfold coordinated structure. The elemental semiconductors germanium $(\mathrm{Ge})$ and silicon $(\mathrm{Si})$ transform from the diamond cubic phase, at ambient pressure, to the metallic $\beta$-tin phase, at pressures of $\sim 10$ and $\sim 12 \mathrm{GPa}$, respectively. ${ }^{2,3}$

Under indentation with a sharp diamond tip, most materials deform plastically by mechanisms such as dislocation slip and/or twinning. Ge and $\mathrm{Si}$, however, have highly covalent bonding and a correspondingly high resistance to dislocation motion. ${ }^{4}$ For these Group IV semiconductors there has been evidence that deformation under indentation may occur by the above-mentioned high-pressure phase transformation. ${ }^{5,6}$ This phenomenon of indentation-induced phase transformation has attracted considerable interest, particularly as the $\beta$-tin phase transforms to metastable phases with distinct electrical properties after unloading. ${ }^{3}$ In diamond-anvil cell studies $\mathrm{Ge}$ transforms from the $\beta$-tin structure (Ge-II) to the st-12 structure (Ge-III) on slow unloading ${ }^{7}$ or the bc 8 structure (Ge-IV) on very fast unloading. ${ }^{8}$

Whereas the indentation-induced phase transformation of $\mathrm{Si}$ has been intensively studied and characterized, ${ }^{9}$ the response of Ge to indentation is less well understood and has generated some controversy. Several studies of Ge using high-load Vickers indenters have observed evidence of a metallic phase transformation: Clarke et al. reported a reversible drop in electrical resistance during indentation and the obser-

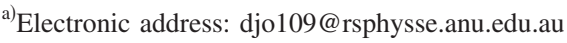

vation by plan-view transmission electron microscopy (TEM) of amorphous material in the residual indent, ${ }^{6}$ and Gogotsi and coworkers observed amorphous $\mathrm{Ge}(a-\mathrm{Ge})$ and $\mathrm{Ge}-\mathrm{III}$ in residual indents by Raman microspectroscopy. ${ }^{10} \mathrm{In}$ an early Vickers study by Gridneva et al., phase transformation was inferred from the temperature-independence of the hardness of Ge below a certain temperature. ${ }^{5}$ Additionally, Pharr et al. observed a resistance drop during nanoindentation, ${ }^{11}$ and one cross-sectional TEM study observed a body-centered cubic (bcc) phase in residual indents. $^{12}$

Other studies employing low-load nanoindentation, however, have failed to find evidence of a high-pressure phase transformation in crystalline Ge. A Raman microspectroscopy study by Gogotsi and coworkers on nanoindents found phase transformation only to occur very rarely and irreproducibly. ${ }^{13}$ A TEM study by Bradby et al. found mechanical twinning to be the dominant deformation mechanism in nanoindents made with a spherical indenter. ${ }^{14}$ Most recently, small amounts of Ge-IV in indents were observed by Jang et al. but could only be reproducibly created using a cube corner indenter, rather than the blunter Berkovich pyramidal indenter. ${ }^{15}$

Furthermore, the nanoindentation load-displacement curve of Ge shows no indications that a phase transformation occurs. The unloading section of the curve for Si generally features a "pop out" (discontinuous depth decrease) or "elbow" (change in slope), depending on unloading conditions. ${ }^{16}$ There is some question as to exactly what these features signify, but it is generally agreed that they are linked to phase transformation behaviors. ${ }^{16,17}$ By contrast, for Ge, the loading section of the curve features multiple small "pop- 


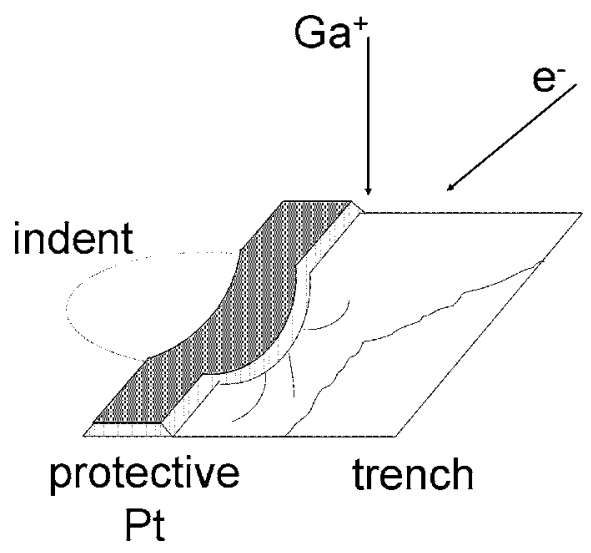

FIG. 1. Schematic of indent cross section milled and viewed in the FIB.

ins" (discontinuous depth increases), but the unloading section is featureless. ${ }^{11,13,14,18}$ One explanation proposed for these divergent results is that phase transformation is only initiated under extreme loading conditions, after severe plastic deformation has occurred; but no direct evidence for such a scenario has been observed.

The indenter used in the present work (UMIS-2000) is capable of applying loads up to $1 \mathrm{~N}$, allowing the investigation of loading regimes associated with Vickers indentation. We focus on the intermediate loading regime to bridge the gap in the literature between the two types of studies, high load and low load. In this regime, novel giant pop-in events are observed, corresponding to several local, and catastrophic, changes in the material, the most notable of which is the presence of an amorphous phase in the residual indent. A range of characterization techniques have been applied, including Raman microspectroscopy, scanning electron microscopy (SEM), focused ion-beam (FIB) cross-sectional analysis, cross-sectional TEM, and optical microscopy. The results point to a deformation scenario involving material removal and lateral cracking, which will be described in the discussion section. The possible mechanisms responsible for the formation of amorphous material will be discussed. Finally, the implications of the findings in terms of previous indentation studies on Ge will be considered.

\section{EXPERIMENTAL PROCEDURE}

Arrays of indents were created in undoped crystalline $\mathrm{Ge}(100)$ with the UMIS-2000 indenter. Two different tip geometries were used: spheroconical $(R \approx 4.3 \mu \mathrm{m})$, and Berkovich. Load was applied and released in a single continuous cycle with no hold period. Tests were also performed in which load was released to $50 \%$ after each loading incre- ment, in order to calculate contact pressure as a function of penetration depth. ${ }^{19}$ Maximum loads ranged from 50 to 1000 $\mathrm{mN}$. Loading rates were $1.0-1.5 \mathrm{mN} \mathrm{s}^{-1}$ and unloading rates were $1.4-1.9 \mathrm{mN} \mathrm{s}^{-1}$, except where otherwise specified.

SEM was conducted using a Hitachi S4500 FESEM operating in secondary-electron mode to investigate the surface crack morphology around indents. A dual ion- and electronbeam FIB microscope (FEI xT Nova Nanolab 200) was used to investigate the subsurface cracking and indent profile. The method of obtaining cross sections through the center of indents was as follows: A layer of platinum was first deposited in the FIB to protect the indent. Then a trench was milled using the $\mathrm{Ga}$ ion beam at $7 \mathrm{nA}$ beam current to expose a vertical cross section. Cleaning mills at lower beam currents were performed to clean the cross section. Finally, the cross section was imaged using the electron beam at a tilt angle of $52^{\circ}$, in secondary-electron mode. A schematic of the crosssectional geometry is shown in Fig. 1.

Raman spectra were collected from the centers of indents with a Raman microscope (Dilor Super LabRam) using a He-Ne laser $(\lambda=632.8 \mathrm{~nm})$. The laser power was kept low $(\sim 100 \mu \mathrm{W})$ to avoid annealing effects. The spot size was $\sim 1 \mu \mathrm{m}^{2}$. Raman spectra were generally taken at least several days after indentation, except where otherwise noted.

A FIB (FEI xP 200) was used to cross-section indents for TEM examination. ${ }^{14} \mathrm{~A} \sim 1 \mu \mathrm{m}$ thick Pt strip was deposited with the FIB to protect the indent during milling. TEM was performed using a Philips CM 300 operating at $300 \mathrm{kV}$.

Optical microscopy was performed on indents using a Leica DM4000 M microscope. Digital micrographs were captured with the attached charge-coupled device (CCD) camera and analyzed with ImageJ (NIH, Bethesda, Maryland) to quantify the amount of debris around each indent. Debris particles showed up against the smooth surface in strong dark contrast. The procedure was as follows: An annular region 40 to $90 \mu \mathrm{m}$ from the indent center was selected, the outer radius limited by the size of the image and the inner radius chosen to exclude the indent itself. The rest of the image was discarded. After thresholding the image, the total area taken up by debris was measured using the particle analysis function. Indents used for debris analysis were created in an array with a $500 \mu \mathrm{m}$ spacing on a clean sample.

\section{RESULTS}

Similar to previously reported studies, ${ }^{11,13,14,18}$ indents with maximum loads of 50 and $100 \mathrm{mN}$ featured multiple pop-ins on loading, each of 10-20 nm extension, and a fea-
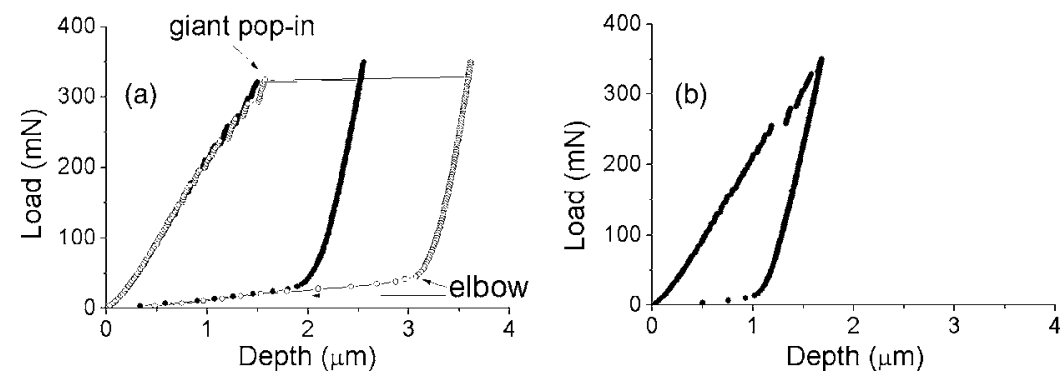

FIG. 2. (a), (b) Force-displacement curves for $350 \mathrm{mN}$ indents in Ge, created using identical test parameters. Curves in (a) feature a giant pop-in event. 


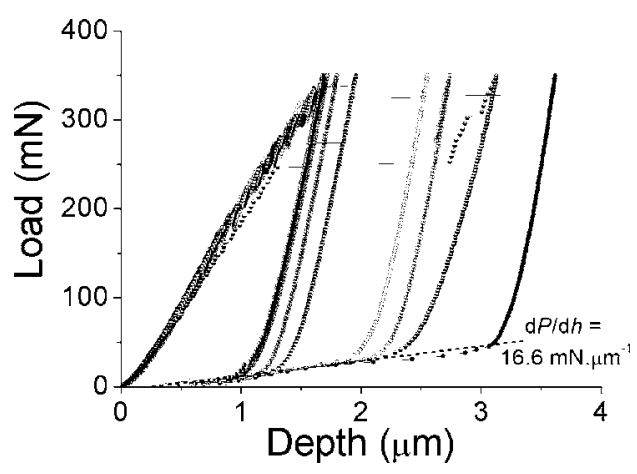

FIG. 3. Force-displacement curves from ten different $350 \mathrm{mN}$ indents in $\mathrm{Ge}$ made with identical test parameters.

tureless unloading curve. With increasing load, more pop-ins were found, increasing somewhat in size (up to $\sim 50 \mathrm{~nm}$ ) at higher loads.

With the spherical tip, at loads upward of $200 \mathrm{mN}$, a giant pop-in of very large magnitude $(>1 \mu \mathrm{m})$ was found to occur. The load at which it occurred varied. Typical examples of the giant pop-in event are shown for $350 \mathrm{mN}$ indents in Fig. 2(a). Indents that featured a giant pop-in consistently also featured a pronounced elbow on unloading, as indicated in Fig. 2(a). The larger the pop-in, the higher the load at which elbowing initiated. A force-displacement curve for an indent made under identical test conditions that did not feature a giant pop-in, is shown for comparison in Fig. 2(b).

Figure 3 shows a number of overlaid $350 \mathrm{mN}$ load forcedisplacement curves. The position and magnitude of the giant pop-in, when it occurs, vary considerably; consequently, the maximum depth also varies. Much less variation is observed in the residual depth at zero load. For most of the indents with a giant pop-in, above the giant pop-in the loading and unloading sections of the curve lie almost atop one another, signifying elastic behavior. Slight elbowing is observed even in curves that do not feature a giant pop-in. The post-elbowing sections of different curves lie closely atop one another, and are highly linear. A linear best fit to the combined post-elbowing sections obtained a slope of $\mathrm{d} P / \mathrm{d} h=16.6 \pm 0.7 \mathrm{mN} \mu \mathrm{m}^{-1}$.

A statistical analysis of the giant pop-in event was conducted using 400 force-displacement curves from $500 \mathrm{mN}$ load spherical indents made with 125 loading increments and 10 unloading increments. $95 \%$ of the indents examined featured a giant pop-in, defined as an extension greater than 600 nm. (Approximately $29 \%$ of the indents featured two or three giant pop-ins: In that event, only the first was used in the analysis.) Histograms of the load at which the giant pop-in was observed and its magnitude are shown in Fig. 4. The average load at which the giant pop-in occurred was $\sim 340 \mathrm{mN}$; the average magnitude of the pop-in was $\sim 2.0 \mu \mathrm{m}$.

To compare indents with, and without, a giant pop-in, statistical analysis was also performed on $350 \mathrm{mN}$ load spherical indents made with 100 loading and 100 unloading increments. Because only $\sim 50 \%$ of the indents featured a giant pop-in, there was considerable variation in the maximum depth at full load. The maximum depth correlated closely with the giant pop-in size $\left(R^{2}=0.96\right)$. Because of elbowing, however, the final depth on complete unloading showed markedly less variation than the maximum depth. Moreover, there was minimal correlation between the final depth and the maximum depth $\left(R^{2}=0.08\right)$.

Typical contact pressure results by the partial unload method $^{19}$ are shown in Fig. 5. The contact pressure prior to the giant pop-in is not constant; rather, it tends to increase steadily until a small pop-in occurs, at which point it drops to a lower value. The giant pop-in occurs, in this case, at a contact pressure of $\sim 9.7 \mathrm{GPa}$; after the giant pop-in, the pressure decreases dramatically. It should be noted that the indenter used in the study is only spherical to a contact depth of $\sim 1.5 \mu \mathrm{m}$; beyond this depth, the Field and Swain analysis will no longer be quantitatively accurate.

Indents made with the Berkovich tip showed multiple small pop-ins. Indents made to loads of $300-500 \mathrm{mN}$ did not feature a giant pop-in, but such a feature was sometimes observed in Berkovich tests at loads upward of $800 \mathrm{mN}$. When a giant pop-in occurred it was accompanied by elbowing on unloading. Berkovich tests without a giant pop-in showed a featureless unloading curve.

The development of surface cracking for spherical indents was investigated by examining residual indents of a range of maximum loads using optical microscopy and SEM. $50 \mathrm{mN}$ indents showed no surface indications of cracking. Indents of maximum load $100 \mathrm{mN}$ and above featured surface traces of radial/median cracking. At loads of 150 and $200 \mathrm{mN}$, some indents showed wing-like features indicative of lateral cracking; at maximum loads of $250 \mathrm{mN}$ and above, lateral cracking was observed around all indents (with or without a giant pop-in).

For loads sufficient for a giant pop-in to occur, indents featured pronounced radial and lateral cracking, as can be seen for the $350 \mathrm{mN}$ indents in Fig. 6. The indent in Fig. 6(a) underwent a giant pop-in; the indent in Fig. 6(b) did not. A number of $350 \mathrm{mN}$ indents were examined by SEM; while
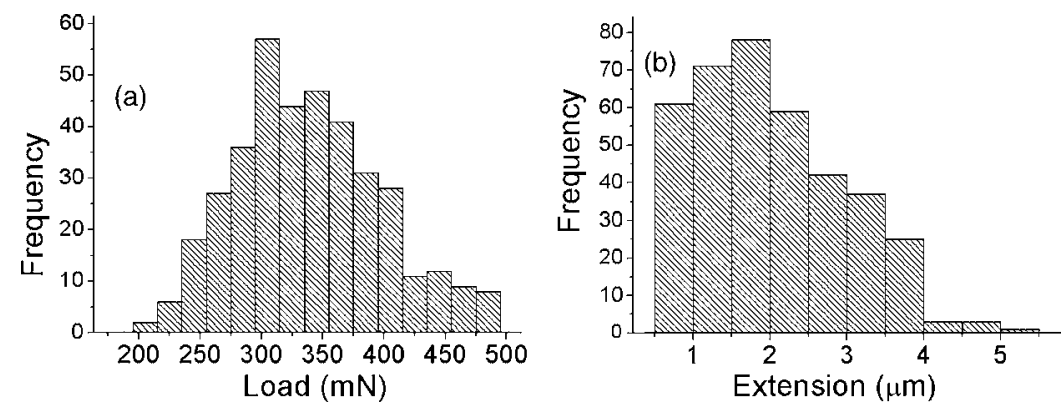

FIG. 4. Histograms of (a) the load at which the giant pop-in occurs, and (b) the magnitude of the giant pop-in for 400 indents made with a spherical tip of radius $4.3 \mu \mathrm{m}$ loaded to $500 \mathrm{mN}$. 

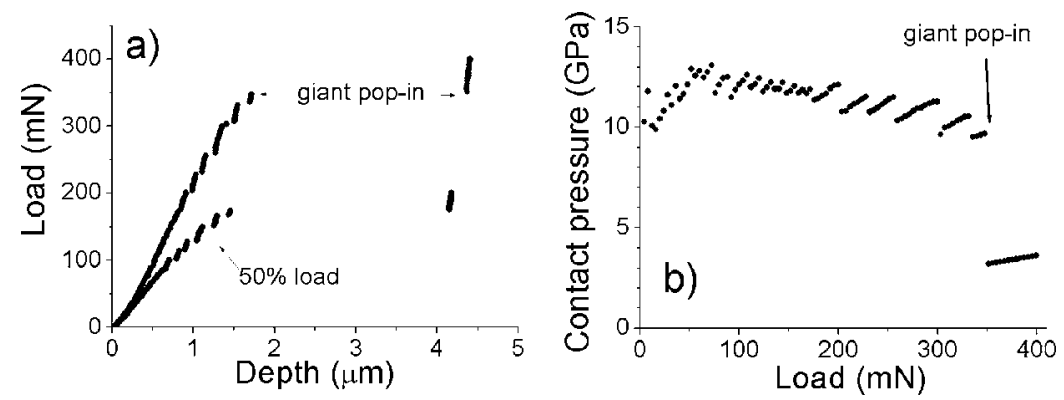

FIG. 5. Partial unload results for Ge. (a) Forcedisplacement data. (b) Contact pressure as a function of load, calculated by the Field and Swain method (Ref. 19).

there was considerable variation in the surface morphology, no obvious marks distinguished indents with a giant pop-in from those without. Lateral cracking generally caused a thin sliver of material around the indent to be partially detached from the bulk, as indicated in Fig. 6(b).

A number of $350 \mathrm{mN}$ indents, both with and without giant pop-in, were cross sectioned and imaged with the dualbeam FIB microscope. Two examples are shown in Fig. 7. Directly beneath indents, extensive crack networks are visible. All indent cross sections revealed large lateral cracks extending outward from the central deformed region. The slivers of detached material above the lateral cracks are uplifted above the bulk surface (dotted line). No distinct phase contrast is visible in either cross section.

The cross sections revealed that indents that had undergone a giant pop-in were significantly deeper than indents which had not. The indent in Figs. $7(\mathrm{c})$ is $\sim 3.2 \mu \mathrm{m}$ deep, whereas the indent in Fig. $7(\mathrm{~d})$ is only $\sim 0.6 \mu \mathrm{m}$ deep. The cross-sectional profile is delineated the boundary between the lighter-colored deposited $\mathrm{Pt}$ and the darker-colored $\mathrm{Ge}$ underneath. The original level of the surface prior to indentation was extrapolated from the level of the undeformed material at the edges of the cross section. The cross-sectional depth was measured from the original level of the surface to the base of the indent. We draw attention to the fact that the cross-sectional depth of the indent that underwent a giant pop-in is considerably greater than the depth at final unload given by the force-displacement curve [Fig. 8(a)] of $0.46 \mu \mathrm{m}$. In contrast, for the indent that did not undergo a giant pop-in, the depth in cross section agrees with the depth at final unload from the force-displacement curve of $0.65 \mu \mathrm{m}$ [Fig. 8(b)]. For the other indents examined, those that had undergone a giant pop-in featured a cross-sectional depth of $1-3 \mu \mathrm{m}$, markedly greater than that indicated by the force-displacement curve. For those with no giant pop-in,
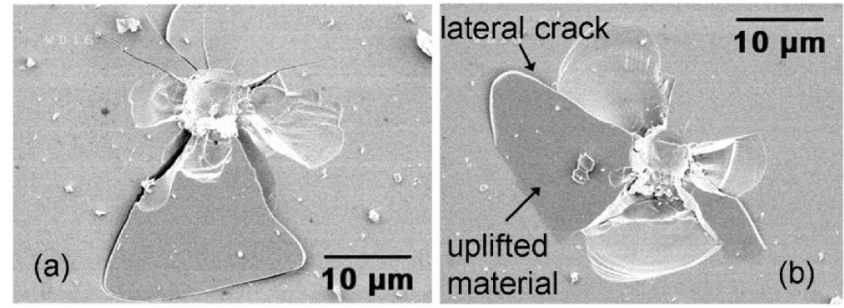

FIG. 6. SEM images of $350 \mathrm{mN}$ load indents: (a) one of the indents in Fig. 2(a) (giant pop-in) and (b) indent in Fig. 2(b) (no giant pop-in). the cross-sectional depth was $<1 \mu \mathrm{m}$, and was in approximate agreement with the force-displacement curve depth at final unload.

Notably, indents with, and without, a giant pop-in differed in the location of the point where the near tip of the lateral crack meets the contact impression. In Fig. 7(c), for the lateral crack on the left, this point lies close to the edge of the contact impression. By contrast, in Fig. 7(d) the lateral crack meets the contact impression close to the base, well below the edge of the contact impression. Other indents examined in cross section were similar: Those with a giant pop-in featured a shallow-initiated lateral crack, while those without a giant pop-in featured only deep-initiated lateral cracks.

The Raman spectrum of Ge-I (diamond cubic) has a single first-order peak at $301 \mathrm{~cm}^{-1}$. The peak broadens and shifts to higher wave numbers $\left(305-310 \mathrm{~cm}^{-1}\right)$ when compressive stresses are present. ${ }^{13}$ Indents at loads up to $200 \mathrm{mN}$ featured only the compressively shifted Ge-I peak in the Raman spectrum, as observed in previous studies. ${ }^{13,14}$ Some
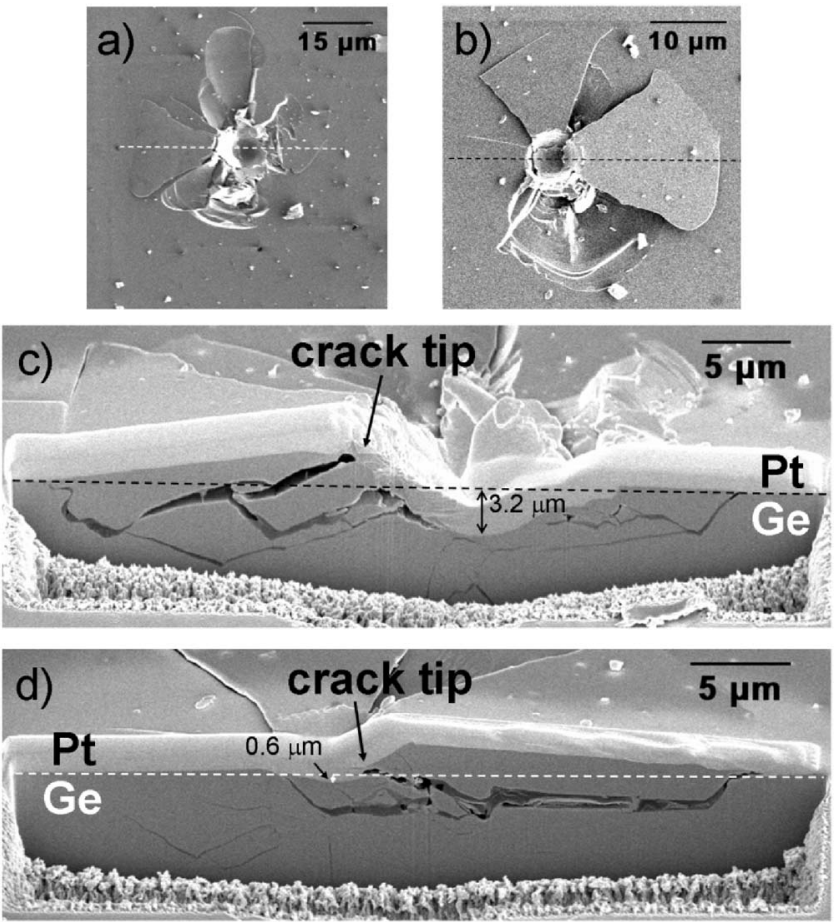

FIG. 7. (a), (b) FIB ion-beam images (prior to Pt deposition) of $350 \mathrm{mN}$ indents: (a) underwent a giant pop-in, (b) did not undergo a giant pop-in. (c) FIB electron-beam cross-sectional image of indent (a). (d) FIB electronbeam cross-sectional image of indent (b). 


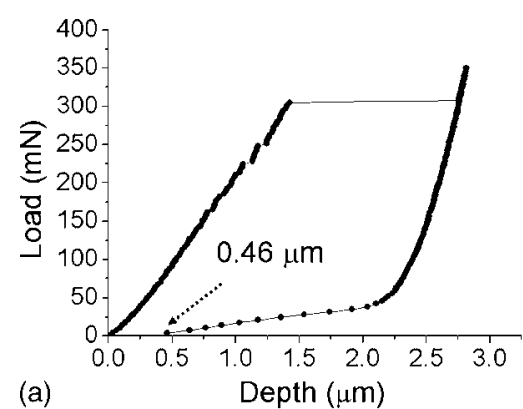

(a)

Depth $(\mu \mathrm{m})$

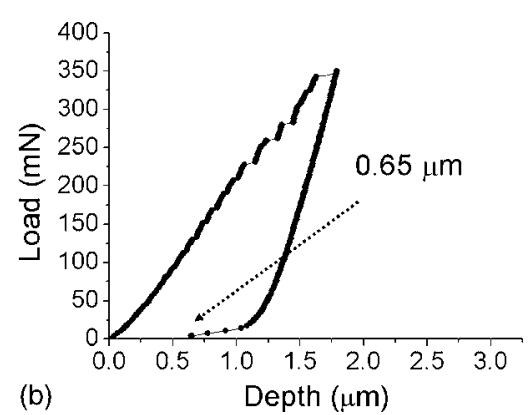

FIG. 8. (a) Force-displacement curve for indent in Fig. 7(a) Force-displacement curve for indent in Fig. 7(b). higher-load indents exhibited a distinctly different Raman spectrum, consisting of a broad, asymmetric band peaking at $280-295 \mathrm{~cm}^{-1}$. It resembles the Raman spectrum of $a-\mathrm{Ge}$, which features a Gaussian band at $\sim 270 \mathrm{~cm}^{-1}$. This type of spectrum was only observed if the indent had undergone a giant pop-in. Typical examples from $350 \mathrm{mN}$ spherical indents are shown in Fig. 9(a). Indents without a giant pop-in featured only the broadened peak at $305-310 \mathrm{~cm}^{-1}$. Such a spectrum is shown in Fig. 9(b).

A number of $350 \mathrm{mN}$ indents were analyzed, 24 in total. Of the ten indents which underwent a giant pop-in, all featured an amorphous Raman spectrum. 13 out of the 14 remaining indents, without a giant pop-in, showed only the Ge-I peak; the last featured additional peaks $(225,242$, and $272 \mathrm{~cm}^{-1}$ ) corresponding to Ge-III (see Discussion).

Raman analysis on Berkovich indents and partial-unload spherical indents yielded similar results: indents with a giant pop-in featured a broad amorphouslike band in the Raman spectrum; other indents featured only the broadened and upshifted Ge-I peak.

A number of high-load indents, with and without a giant pop-in, were examined by Raman spectroscopy within three hours of indentation. The same correlation of the amorphous phase with the giant pop-in was noted. No extra peaks were observed.

To examine the effect of the unloading rate, sets of 350 $\mathrm{mN}$ indents were made using slow unloading rates of 0.71 and $0.34 \mathrm{mN} \mathrm{s}^{-1}$. Both sets of indents showed similar elbowing behavior in the force-displacement curves as observed for faster unloading rates. Raman analysis on these indents again found that those with a giant pop-in featured an amorphous band, with no extra peaks observed; while those indents without a giant pop-in featured only the broadened and upshifted Ge-I peak.

A recent report that Ge-III is unstable under Raman laser irradiation (Ref. 20) has caused uncertainty in other indentation studies of Ge. ${ }^{15,21}$ We addressed this by examining powder-form Ge-III/Ge-I (prepared in a high-pressure cell and structure confirmed by x-ray diffraction) with the Raman instrument used in the study. We found that the Ge-III annealed very rapidly at a laser power of $1 \mathrm{~mW}$; however, at $0.1 \mathrm{~mW}$ or below, a stable spectrum could be recorded, as shown in Fig. 10, with sharp peaks at 227, 243, 272, and $298 \mathrm{~cm}^{-1}$. The $298 \mathrm{~cm}^{-1}$ peak probably belongs to Ge-I (or hexagonal diamond $\mathrm{Ge}-\mathrm{V})$; the other peaks belong to Ge-III. ${ }^{10,22}$ This shows that Ge-III is stable under Raman analysis if a sufficiently low laser power is used, as in this study.

Previous indentation studies have used TEM analysis of cross-sectional samples prepared by FIB in order to investigate subsurface phase transformation and other deformation mechanisms. ${ }^{14,23}$ Indents to the loads of interest, $300 \mathrm{mN}$ and above, were quite difficult to examine in this way: Attempts to prepare a cross section of $\sim 100 \mathrm{~nm}$ thickness were frustrated by high-residual stresses and extensive cracking beneath the indents, which usually caused the cross section to disintegrate during preparation. An intact cross section obtained from a $400 \mathrm{mN}$ indent, without a giant pop-in, is shown in Fig. 11. A high degree of damage is revealed. Thick

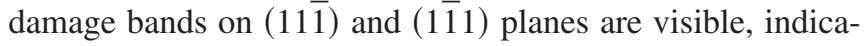
tive of twinning or slip. These extend $\sim 4 \mu \mathrm{m}$ below the surface. Many of the damage bands terminate at the point where they meet another band on the opposing $\{111\}$ plane. Numerous cracks (bright features) are visible. Several of the cracks initiate at the intersection of two damage bands on opposing $\{111\}$ planes. One such crack is labeled in Fig. 11. Selected-area diffraction on the deformed region below the indent showed streaky reflections indicative of twinning, as previously observed in $\mathrm{Ge},{ }^{14}$ but no signs of amorphization or other phase transformations, consistent with the Raman microspectroscopy results.

Microscopy revealed high-load indents in Ge to be surrounded by considerable amounts of scattered debris. Indents that had undergone a giant pop-in featured a greater quantity of debris. Example optical micrographs of $350 \mathrm{mN}$ indents (with and without a giant pop-in) are shown in Figs. 12(a)

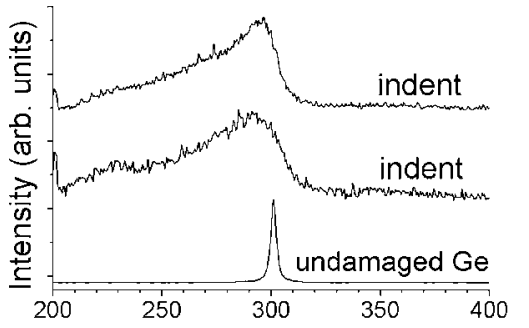

(a)

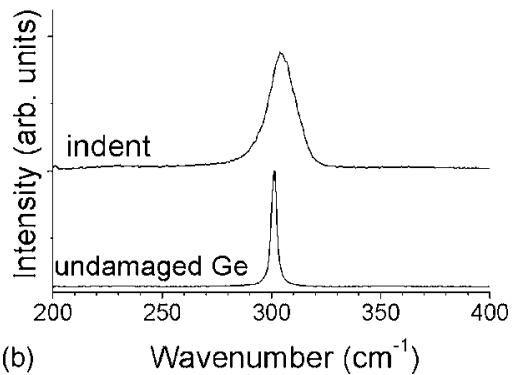

(b)
FIG. 9. (a) Raman spectra from indents in Fig. 2(a) (giant pop-in), with spectrum from undamaged Ge for comparison, (b) Raman spectrum from indent in Fig. 2(b) (no giant pop-in). 
and 12(b). The amount of debris was quantitatively measured, according to the method described in the Experimental Procedure. A number of indents were analyzed in this way. For each indent, the total area of debris was compared with the size of the largest pop-in in the force-displacement curve (either a giant pop-in or a smaller pop-in). The results are shown in Fig. 13(a). Quantity of debris showed a clear correlation $\left(R^{2}=0.68\right)$ with pop-in size.

Larger debris particles ( $>1 \mu \mathrm{m}$ in width) around indents with a giant pop-in were analyzed by Raman spectroscopy. For all particles the Ge-I peak was observed at $\sim 300 \mathrm{~cm}^{-1}$, without additional bands.

\section{DISCUSSION}

It is notable that multiple small pop-ins were observed at all stages of loading. The main causes of pop-in in monolithic materials are phase transformation, ${ }^{17,24}$ bursts of dislocation activity, ${ }^{25-27}$ and cracking. ${ }^{28}$ The underlying common factor is an energy barrier hindering inelastic deformation: For example, the energy required to nucleate dislocations in pristine crystal, ${ }^{25}$ or to initiate a crack. ${ }^{28}$ This barrier leads to increased elastic strain. When the barrier is overcome, the accumulated strain energy is rapidly converted to inelastic deformation. The associated increase in indenter penetration is the observed pop-in.

The TEM cross section in Fig. 11 offers an explanation as to why pop-in is so prevalent in Ge. It shows numerous pinned dislocation/twinning bands, as was also observed on a smaller scale in low-load indents. ${ }^{14}$ When pinning occurs, further propagation of the pinned slip or twinning system is impeded, arresting plasticity and so increasing elastic strain. When a fresh slip or twin band nucleates it will propagate deeply into the material under the action of the built-up strain energy, resulting in pop-in. This sequence of accumulation of strain, followed by release, is reflected in the tendency of the contact pressure in Fig. 5(b) to increase, prior to a pop-in, and drop sharply, after the pop-in. The local stress concentration induced by pinning can also initiate a crack, ${ }^{29}$ as observed in the TEM cross section. Depending on its location and orientation, the initiated crack may propagate to relieve strain, again causing pop-in.

The giant pop-in is clearly a dramatic damage event, in which the instantaneous indenter penetration frequently exceeds total elastic-plastic penetration. The pinning mechanism described for the smaller pop-ins is insufficient to account for its magnitude. Examining the force-displacement curve alone, in which the pop-in penetration is apparently recovered by elbowing on unloading, one might attribute the giant pop-in to a reversible mechanism. One such mechanism would be the sudden transformation of a large volume of material beneath the indenter tip to metallic Ge-II, which is $\sim 20 \%$ more dense than Ge-I. ${ }^{2}$ The transformation on unloading of Ge-II to $a$-Ge, which at ambient pressure is close in density to Ge-I, ${ }^{30}$ would then account for the elbowing.

For a pop-in of $1-2 \mu \mathrm{m}$ to occur by this mechanism, the transformation of a volume at least 5-10 $\mu \mathrm{m}$ deep would be required. Calculations of the Hertzian stress distributions beneath the tip at the loads of interest $(300-400 \mathrm{mN})$ were

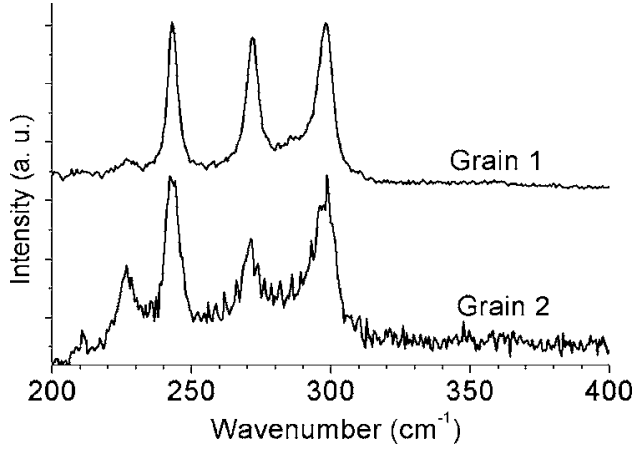

FIG. 10. Raman spectra from powder-form Ge-III/Ge-I prepared in a diamond-anvil cell

made using the Elastica software package (ASMEC, Germany). The calculated hydrostatic pressure exceeded the threshold pressure for transformation (7-8 $\mathrm{GPa}^{31}$ ) to a depth of only about $2 \mu \mathrm{m}$. The analysis neglects plastic deformation, which would lower the pressure further still. The giant pop-in therefore cannot be solely attributed to the sudden metallic phase change of a large volume of material.

Furthermore, cross sectioning (Fig. 7) reveals that indents featuring a giant pop-in are markedly deeper than indents without a giant pop-in. This implies that the giant pop-in involves material removal. Quantities of scattered debris are observed around indents with a giant pop-in, the amount of debris correlating with the size of the pop-in. It is reasonable to suppose that the material missing from the indent is the same material that is observed as debris. Thus we propose that the giant pop-in in the force-displacement curve is caused by material being ejected violently from the deformation zone beneath the indenter tip. The tip sinks into the space left by the removed material, causing the observed sudden increase in depth.

Indents with a giant pop-in were distinguished by lateral cracks initiated just below the sample surface, at the periphery of the deformation zone. It seems probable that these

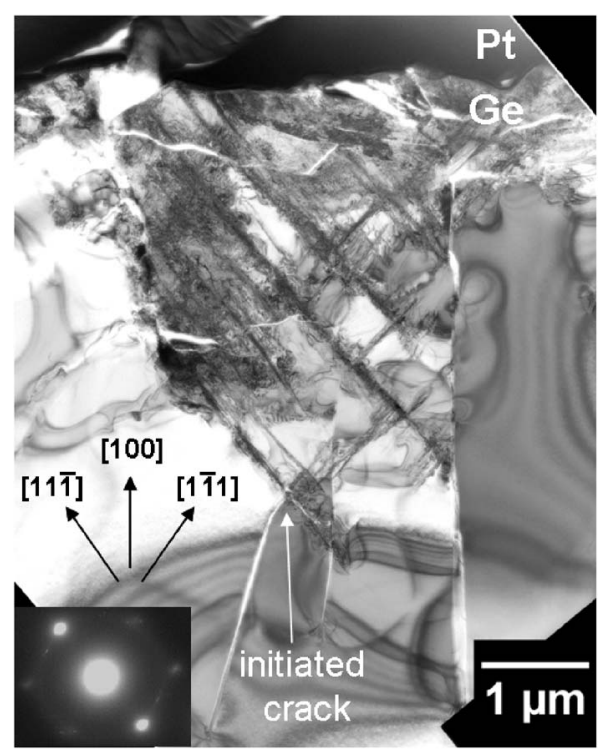

FIG. 11. Cross-sectional bright-field TEM of a $400 \mathrm{mN}$ indent without a giant pop-in. Inset: diffraction pattern from deformed region. 


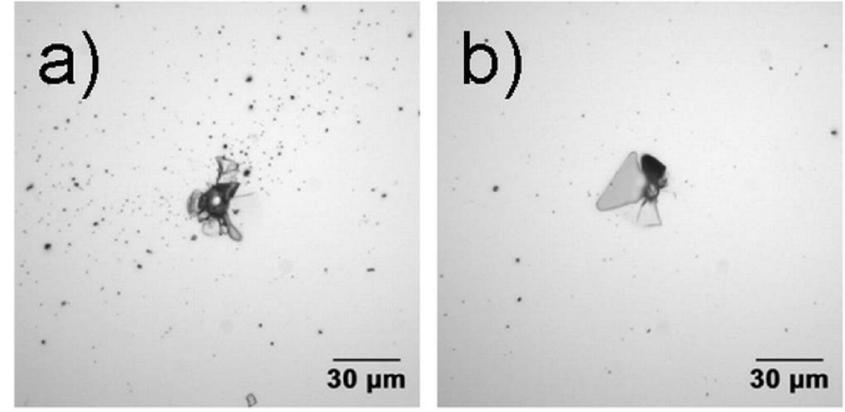

FIG. 12. Optical micrographs of $350 \mathrm{mN}$ indents: (a) with a large maximum pop-in $(1.96 \mu \mathrm{m})$, (b) with a small maximum pop-in $(0.17 \mu \mathrm{m})$.

shallow laterals allow the giant pop-in to occur by enabling material to escape from the central deformation zone. The opening of the cracks on loading releases the material beneath the indenter from confinement, allowing it to be ejected to the free surface. Indentation-induced lateral cracks propagate under the effect of tensile stresses approximately normal to the free surface $\left(\sigma_{z z}\right)$, induced by plastic deformation. ${ }^{32}$ Lateral cracking is favored on load release, when these stresses reach a maximum. ${ }^{32}$ However, in crystalline materials, lateral cracking has also been observed to occur during loading. ${ }^{33}$ The maximum $\sigma_{z z}$ stress occurs at a depth roughly equal to the contact radius, ${ }^{34}$ at a radial position corresponding to the boundary of the plastic zone. ${ }^{32,34}$ Within the plastic zone, $\sigma_{z z}$ becomes compressive.

Cracks that initiate at shallow depths will be able to open on loading, because the tip of the crack closest to the contact impression is only weakly confined by the indenter, and they can therefore propagate up to the free surface. Cracks that initiate at lower depths will only open on unloading: during loading, the crack tip will be confined below the indenter, effectively trapped in the induced compressive stress field. Consequently, only shallow-initiated lateral cracks will be able to contribute to the giant pop-in, in agreement with the observations.

Much greater loads were required to initiate a giant pop-in when a Berkovich indenter was used, than when the

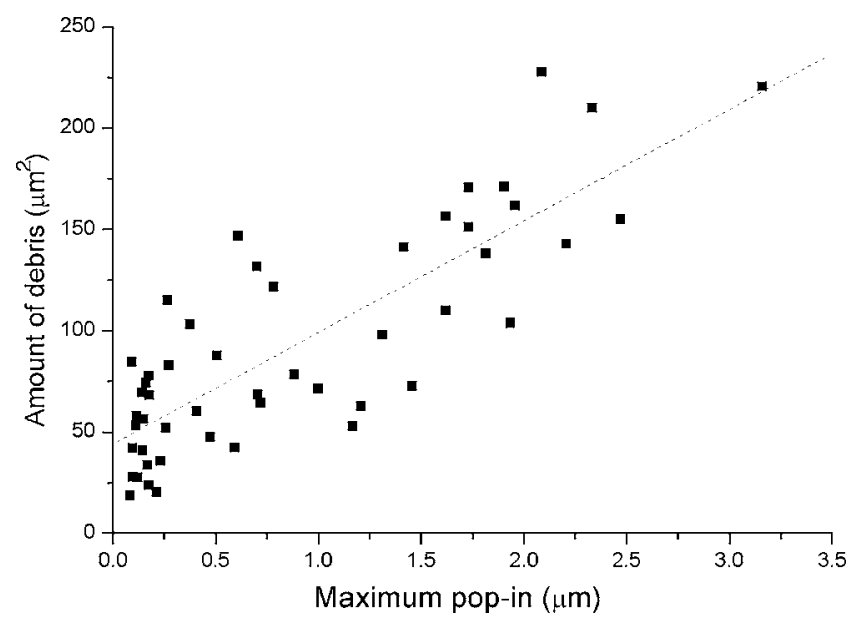

FIG. 13. The amount of debris around $350 \mathrm{mN}$ indents plotted against the size of the largest pop-in for the indent. The dotted line is a guide for the eye. spherical indenter was used. We note that for the Berkovich indenter, which is self-similar, the induced strain is approximately constant, whereas for the spherical tip the strain increases continuously with penetration depth, acting to promote cracking and thus favor the occurrence of the giant pop-in. Additionally, the sharp corners of the Berkovich indenter act as stress concentrators to induce radial/median cracking, ${ }^{28}$ presumably at the expense of the lateral cracking which is responsible for the giant pop-in. A study of the giant pop-in using a range of tip geometries would shed further light on the importance of these factors.

Most interestingly, indents featuring a giant pop-in consistently contained $a$-Ge, whereas other indents contained only the original Ge-I phase. We note that if a lateral crack opens during loading, the contact area supporting the indenter is likely to decrease. With the contact area decreased, and the load unchanged, the contact pressure must necessarily increase. We note also that the pressure increase would be transient: The increase in penetration depth after the material removal event would mean a greatly increased contact area, and consequently a diminished pressure. It seems most likely that these rapid pressure changes during the giant pop-in are responsible for creating the observed amorphous material. One possibility is that a high-pressure metallic phase $\left[\mathrm{Ge}-\mathrm{II},{ }^{2}\right.$ or possibly high-density $a$-Ge (Ref. 35)] forms on pressure increase; the subsequent rapid pressure decrease would act to quench the metallic material to an amorphous structure, as occurs in Si when load is rapidly released. ${ }^{16,23}$ The other possibility is a direct transformation from Ge-I to $a$-Ge. Although there is little evidence for this type of behavior in previous indentation studies, it is known that a high density of crystalline defects can induce amorphization, by raising the free energy of the lattice. Such defect-induced amorphization is observed, for example, under high-fluence ion implantation of semiconductors. ${ }^{36}$ As can be seen in the TEM image in Fig. 11, the defect density beneath the indent is high, and might increase further in response to a sudden increase in contact pressure, making a direct Ge-I to $a$-Ge transformation favorable. The evidence at hand does not allow either of these scenarios to be conclusively ruled out, nor does it preclude a different or more complex sequence of events, leading to the formation of $a$-Ge.

If the giant pop-in is due to physical removal of material, an inherently irreversible process, the task remains to explain the apparent depth recovery observed through elbowing. The shape of the force-displacement curve, post-elbowing (Fig. 3 ), indicates a highly linear response, with a much lower compliance than the bulk material. Such a response is expected from plates of material around the indent detached by lateral cracking. The detached plates can be modeled as loaded cantilevers. ${ }^{37}$ Using a quarter-plate approximation for the crack configuration and assuming uniform crack length and depth, the stiffness of the plates $k$ is given by ${ }^{37}$

$$
k=4 E h^{3} / 3 c^{2},
$$

where $E$ is the Young's modulus, $h$ is the crack depth, and $c$ is the crack length. Inserting a Young's modulus of 132 $\mathrm{GPa}^{38}{ }^{38}$ and typical crack dimensions, measured from $350 \mathrm{mN}$ indents of $c=17 \mu \mathrm{m}$ and $h=2.5 \mu \mathrm{m}$ into Eq. (1), gives a 

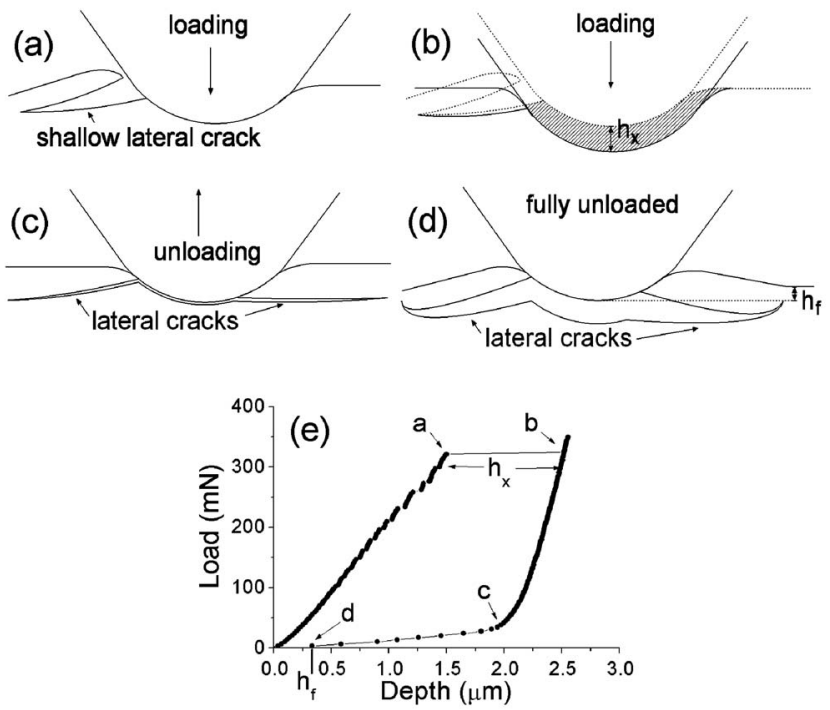

FIG. 14. Schematic representation of the deformation process. (a) A shallow lateral crack opens, which (b) triggers material removal (shaded area), causing the indenter depth to increase by $\mathrm{h}_{x}$. Initial unloading occurs by elastic recovery in the bulk. (c) When bulk recovery is complete, the tip loses contact with the base of the indent. Beyond this point, the force on the tip is due to lateral plates of material, until the tip is fully unloaded [diagram (d)] (d) Points on the force-displacement curve corresponding to the diagrams.

stiffness of $k=9.5 \mathrm{mN} \mu \mathrm{m}^{-1}$. Given the simplifying geometrical assumptions made, this is in reasonable agreement

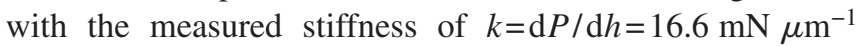
from Fig. 3.

It can be seen that even indents without a giant pop-in show some slight elbowing [e.g., Fig. 2(b)]. The elbowing after the giant pop-in seems to be the same response, only initiating earlier on unloading (that is, at a higher load) and occurring to a much greater extent. Initially during unloading, depth recovery occurs by relaxation in the bulk. Only when most of the bulk elastic strain is released will the compressive stresses diminish sufficiently for the crack to open. The removal of material during the giant pop-in releases a considerable amount of stored elastic strain energy. Consequently, after the giant pop-in, full bulk recovery is completed at an earlier stage of unloading. After bulk recovery is completed, elbowing begins. At this point the indenter will actually lose contact with the bulk, only remaining in contact with the plates detached by lateral cracking. The subsequent force-displacement response, as well as the final depth after complete unloading, are governed by the uplift of these lateral plates. A schematic of this full sequence of events is given in Fig. 14.

The Ge-III spectrum was observed in a very small number of indents out of the many examined. In each case, the maximum load was relatively high $(>300 \mathrm{mN})$, and the indent had not undergone a giant pop-in. Since the parameters leading to reproducible Ge-III formation could not be established, no general conclusions can be drawn from the observations. In $\mathrm{Si}$, the unloading rate largely determines whether the metallic phase reverts to amorphous or crystalline phases, ${ }^{16}$ however, unload rates ranging from 1.9 to
$0.34 \mathrm{mN} \mathrm{s}^{-1}$ were used in this study and found to have no effect on the Raman spectra observed in the residual indent.

We have found in this study that phase transformations occur under high-load indentation that are absent at lower loads. This offers a possible explanation for the discrepancy between previous investigations utilizing high-load Vickers indentation, which observed a phase transformation in $\mathrm{Ge},{ }^{6,10}$ and those utilizing low-load nanoindentation, which did not. ${ }^{13,14} \mathrm{~A}$ giant pop-in would not be detected in a Vickers test, in which the only quantity measured during testing is the maximum applied load. The maximum loads in the highload studies were $500 \mathrm{mN}$ or more, suggesting that a giant pop-in event may have occurred, although the likelihood of giant pop-in under a Vickers tip geometry remains to be investigated. We note also that typical loading rates in a Vickers test are a few orders of magnitude greater than loading rates achievable with a nanoindentation instrument, which is likely to be a significant factor.

\section{CONCLUSION}

The deformation behavior of crystalline Ge under indentation has been studied over a wide load range. At low loads, deformation occurs via shear-induced slip and mechanical twinning. As load increases, cracks develop, nucleated at the intersections of shear-damage bands. At a certain critical load, a dramatic material removal event occurs, in which material is forcefully ejected from the central zone of deformation beneath the indenter. This gives rise to a very large pop-in feature in the indentation force-displacement curve. The material removal event appears to be triggered by the development of a shallow lateral crack immediately adjacent to the indenter, which releases the compressed material beneath the indenter from confinement.

The material removal event relieves a significant fraction of the elastic strain induced by indentation. Consequently, during unloading, before load is fully released, bulk elastic recovery reaches completion and lateral crack opening commences. This gives rise to elbowing in the forcedisplacement curve. The linear and compliant post-elbowing response is in agreement with the predicted behavior of plates of material detached by lateral cracks.

Interestingly, after the giant pop-in, residual indents contain an amorphous-like structural phase. This phase is most likely a result of the sudden pressure changes associated with the giant pop-in. It is possible that the amorphous material is the product of a high-pressure metallic phase transformation, but it is also possible that it transforms directly from the original crystalline phase, through a defect-induced process. Below the critical load for giant pop-in, the material in the residual indent is untransformed from the Ge-I phase. This suggests that conflicting reports on the indentation behavior of crystalline Ge may be partially attributable to differences in maximum load between studies. The giant pop-in we have observed under large-scale deformation in Ge triggers a phase transformation to $a$-Ge that is absent at smaller scales.

${ }^{1}$ G. J. Ackland, Rep. Prog. Phys. 64, 483 (2001).

${ }^{2}$ J. C. Jamieson, Science 139, 762 (1963).

${ }^{3}$ J. Crain, G. J. Ackland, and S. J. Clark, Rep. Prog. Phys. 58, 705 (1995). 
${ }^{4}$ J. J. Gilman, Philos. Mag. B 67, 207 (1993).

${ }^{5}$ I. V. Gridneva, Y. V. Milman, and V. I. Trefilov, Phys. Status Solidi A 14 177 (1972)

${ }^{6}$ D. R. Clarke, M. C. Kroll, P. D. Kirchner, R. F. Cook, and B. J. Hockey, Phys. Rev. Lett. 60, 2156 (1988).

${ }^{7}$ F. P. Bundy and J. S. Kasper, Science 139, 340 (1963).

${ }^{8}$ R. J. Nelmes, M. I. McMahon, N. G. Wright, D. R. Allan, and J. S. Loveday, Phys. Rev. B 48, 9883 (1993).

${ }^{9}$ V. Domnich and Y. Gogotsi, Rev. Adv. Mater. Sci. 3, 1 (2002).

${ }^{10}$ A. Kailer, K. G. Nickel, and Y. G. Gogotsi, J. Raman Spectrosc. 30, 939 (1999).

${ }^{11}$ G. M. Pharr, W. C. Oliver, R. F. Cook, P. D. Kirchner, M. C. Kroll, T. R. Dinger, and D. R. Clarke, J. Mater. Res. 7, 961 (1992).

${ }^{12}$ S. J. Lloyd, J. M. Molina-Aldareguia, and W. J. Clegg, J. Mater. Res. 16, 3347 (2001).

${ }^{13}$ Y. G. Gogotsi, V. Domnich, S. N. Dub, A. Kailer, and K. G. Nickel, J. Mater. Res. 15, 871 (2000).

${ }^{14}$ J. E. Bradby, J. S. Williams, J. Wong-Leung, M. V. Swain, and P. Munroe, Appl. Phys. Lett. 80, 2651 (2002).

${ }^{15}$ J. Jang, M. J. Lance, S. Wen, and G. M. Pharr, Appl. Phys. Lett. 86, 131907 (2005).

${ }^{16}$ V. Domnich, Y. Gogotsi, and S. N. Dub, Appl. Phys. Lett. 76, 2214 (2000).

${ }^{17}$ E. R. Weppelmann, J. S. Field, and M. V. Swain, J. Mater. Res. 8, 830 (1992).

${ }^{18}$ S. V. Hainsworth, A. J. Whithead, and T. F. Page, in Plastic Deformation of Ceramics, edited by R. C. Bradt, C. A. Brookes, and J. L. Routbort (Plenum, New York, 1995), p. 173

${ }^{19}$ J. S. Field and M. V. Swain, J. Mater. Res. 8, 297 (1993).

${ }^{20}$ H. Olijnyk and A. P. Jephcoat, Phys. Status Solidi A 211, 413 (1999).
${ }^{21}$ G. Patriarche, E. Le Bourhis, M. M. O. Khayyat, and M. M. Chaudhri, J. Appl. Phys. 96, 1464 (2004).

${ }^{22}$ R. J. Kobliska, S. A. Solin, M. Selders, R. K. Chang, R. Alben, M. F. Thorpe, and D. Weaire, Phys. Rev. Lett. 29, 725 (1972).

${ }^{23}$ J. E. Bradby, J. S. Williams, J. Wong-Leung, M. V. Swain, and P. Munroe, Appl. Phys. Lett. 77, 3749 (2000).

${ }^{24}$ J. E. Bradby, J. S. Williams, and M. V. Swain, Phys. Rev. B 67, 085205 (2003).

${ }^{25}$ D. Lorenz, A. Zeckzer, U. Hilpert, P. Grau, H. Johansen, and H. S. Leipner, Phys. Rev. B 67, 172101 (2003).

${ }^{26}$ J. E. Bradby, J. S. Williams, and M. V. Swain, J. Mater. Res. 19, 380 (2004).

${ }^{27}$ H. Bei, E. P. George, J. L. Hay, and G. M. Pharr, Phys. Rev. Lett. 95, 045501 (2005).

${ }^{28}$ J. S. Field, M. V. Swain, and R. D. Dukino, J. Mater. Res. 18, 1412 (2003).

${ }^{29}$ A. N. Stroh, Proc. R. Soc. London, Ser. A 223, 404 (1954).

${ }^{30}$ T. B. Light, Phys. Rev. Lett. 22, 999 (1969).

${ }^{31}$ C. S. Menoni, J. Z. Hu, and I. L. Spain, Phys. Rev. B 34, 362 (1986).

${ }^{32}$ S. S. Chiang, D. B. Marshall, and A. G. Evans, J. Appl. Phys. 53, 298 (1982).

${ }^{33}$ R. F. Cook and G. M. Pharr, J. Am. Ceram. Soc. 73, 787 (1990).

${ }^{34}$ X. Chen, J. W. Hutchinson, and A. G. Evans, J. Am. Ceram. Soc. 88, 1233 (2005).

${ }^{35}$ M. Durandurdu and D. Drabold, Phys. Rev. B 66, 041201 (2002).

${ }^{36}$ J. W. Corbett, J. P. Karins, and T. Y. Tan, Nucl. Instrum. Methods 182183, 457 (1981)

${ }^{37}$ D. B. Marshall, B. R. Lawn, and A. G. Evans, J. Am. Ceram. Soc. 65, 561 (1982).

${ }^{38}$ J. J. Wortman and R. A. Evans, J. Appl. Phys. 36, 153 (1965). 